\title{
Differences between a birth trauma and compression hypoxia
}

\section{Vasily Vlasyuk}

Department of forensic medicine of the S. M. Kirov Military Medical Academy, Saint-Petersburg, Russia

*Corresponding Author : Vasily Vlasyuk, Department of forensic medicine of the S. M. Kirov Military Medical Academy, Saint-Petersburg, Russia. E-mail: vasily-vlasyuk@yandex.ru

Received date: July 23, 2019; Accepted date : August 27, 2019; Published date: September 17, 2019

Citation : Vasily Vlasyuk. (2019) Differences between a birth trauma and compression hypoxia. J Neuroscience and Neurological Surgery. 4(4);

Doi: 10.31579/2578-8868 /081

Copyright : (c) 2019 Vasily Vlasyuk. This is an open-access article distributed under the terms of The Creative Commons Attribution License, which permits unrestricted use, distribution, and reproduction in any medium, provided the original author and source are credited.

\section{Abstract}

For a long time there is a problem of separation of hypoxia (asphyxia) and birth trauma. To resolve it, it is necessary to recognize this type of lesion as "compression hypoxia" and share it with a birth trauma. Morphological signs of differences between compression hypoxia and birth trauma, two forms of lesions caused by head compression, are given. The causes of death in birth trauma of the skull and the causes of intrapartum hypoxia are described. It has been shown that in many intranatally deceased children and newborns, intradural hemorrhages in the cerebellum are known, indicating head compression during labor.

Key words: intrapartum hypoxia, compression of the head, configuration of the head (molding), compression hypoxia, birth

\section{Introduction}

It has long been a discussion among pathologists and clinicians about the differences between birth trauma and hypoxia (asphyxia). What damages in newborns to carry to birth trauma, and what - hypoxia? The outstanding American pediatric pathologist, E. Potter, pointed out the importance of separating hypoxic and traumatic lesions [1], but to date these issues have not been resolved. At the present time, the diagnosis of "hypoxic-ischemic encephalopathy" (HIE) is widespread. At the same time, "cranial-brain compression ischemic encephalopathy" [2,3] is distinguished, which includes brain damage caused by compression of the skull during labor (with a head configuration, using obstetric forceps, a vacuum extractor and other causes). There is the concept of "fetal head compression syndrome" [4], where it considered predominantly gross traumatic damages to the skull and brain. There is an idea that birth trauma can be mechanical and hypoxic [5]. M.Gutner [6] considered the leading hypoxia of the fetus to be "circulatory hypoxia", to which he attributed brain damage caused by impaired blood circulation associated with compression of the head of the fetus during childbirth. All this indicates the existence of the problem of differential diagnosis of hypoxic and traumatic lesions. The question arises whether all the damage caused by compression of the skull (mechanical forces) should be attributed to birth trauma? Can compression lead to hypoxia of the fetus and its brain? Both in the domestic and foreign literature recognize the existence of brain damage to the fetus and newborn, caused by the configuration of the head, compression of the skull, hypoxia, caused by venous congestion of the blood in expressed configuration of the head. Can this condition be attributed to birth trauma, since it is caused by the action of mechanical forces in the process of labor? Is it correct to attribute this condition to hypoxia, since the child has no gross mechanical damage to the skull and brain? To answer the questions it is necessary to identify the differences between birth trauma and hypoxia.

\section{Material and method}

The study was conducted on non-selective material of 344 intranatally dead fetuses and newborns. A pathologic-anatomical study with a detailed study of the cerebellar tentorium, brain and spinal cord was performed. The study was conducted in accordance with its own method of opening the skull and extracting the brain [7]. For the purpose of differential diagnosis of hypoxic-ischemic lesions caused by circulatory hypoxia and birth trauma, detailed morphological studies of the skull, head tissues, bone displacement,

changes in the sinuses, dura mater, and brain were carried out.
Birth trauma as the main disease with various ruptures and subdural hemorrhages was detected in 49 children (14.2\%). In 7 cases (2\%), small ruptures of the cerebellar structure were found without subdural hemorrhage. In these cases, the existing birth injury is a concomitant or underlying disease. In 35 children (10.2\%) hemorrhages in the cerebellum were discovered, caused by its overstretching during labor. Thus, $24.4 \%$ of children experienced compression (physical) effects on the skull during childbirth, half of which were diagnosed with birth injury. In 13 children with intradural hemorrhages, aspiration of amniotic fluid was detected (37.1\%), confirming the development of intranatal hypoxia in them, and in 11 premature infants $(31.4 \%)$ there was RDS-syndrome (pneumopathy). Consequently, more than half of the children $(68.5 \%)$ with intradural hemorrhages in the cerebellar model died from pulmonary pathology.

Intranatal hypoxia as a cause of death was detected in 45 children (13\%). Its causes were violations of the uteroplacental circulation (11 cases), especially premature placental abruption, weakness of labor activity (3 cases), pathology of the umbilical cord (3 cases), cardiac arrest of the mother ( 1 case), dystocia, etc. In 14 cases the cause of intranatal hypoxia, even after careful study of the after birth, was unclear $(31.1 \%)$. In the study of the brain, subarachnoid and subpial hemorrhages were found in 9 children, hemorrhages in the cerebellum in 4, subependymal and intraventricular hemorrhages in 3, and in 6 children intradural hemorrhages in the cerebellum were found. These data indicate that in some children with intranatal hypoxia, there was compression of the brain with the development of hemorrhages in the tentoria, subarachnoidal, subpial, and other hemorrhages. It was possible to assume that intranatal hypoxia in unclear cases could be caused by compression of the skull.

The results of the study of causes of death indicate the existence of birth trauma (1), hypoxia (2) caused by impaired uteroplacental circulation, pathology of the umbilical cord and diseases of the mother, as well as conditions (3) in which children have slight signs of mechanical (compression) damage in childbirth, which are combined with signs of pronounced hypoxic lesions of brain neurons. This condition can be related to "compression hypoxia" (CH). Traumatic injuries (4) that occur during childbirth, which are concomitant or background lesions, should also be highlighted.

Since both birth trauma and circulatory hypoxia are caused by the action of mechanical forces, these damages should be distinguished. In order to clearly distinguish between birth trauma and hypoxia, it is necessary to distinguish such a type of hypoxia as $\mathrm{CH}$ [8], and to clearly know the damage characteristic of a given brain lesion. In view of the material it is possible to carry out differentiation lineages arising from damage caused by compression of the skull, as follows.

The following major mechanical damages are attributable to birth trauma (BT) of the skull [7]:

1) ruptures of the tentorium cerebelli, falx, bridging veins, tributaries of the vein of Galen, 
2) subdural and epidural hemorrhages,

3) subarachnoid hematomas,

4) large focal intracerebral and intracerebral hemorrhages,

5) compression portions on the surface of the brain,

6) cephalohematomas

7) fractures of the skull.

Mechanical damages related to $\mathrm{CH}$ of the skull and brain are:

1) intradural hemorrhages in the tentorium cerebelli and falx cerebry,

2) area of periosteal stagnation of blood of the skull,

3) spotty and pinpoint epidural hemorrhages,

4) spotted subpial hemorrhages on the tops of the convolutions, caused by the rupture of small vessels and capillaries between the pia mater and the molecular layer of the cortex,

5) subarachnoid hemorrhage in areas of outflow of the bridging veins caused by rupture of the tributaries of the bridging veins,

6) small focal intracerebral hemorrhages in areas of pressure on the brain of the bones of the skull,

7) intraventricular hemorrhages due to rupture of the capillaries of the vascular plexuses of the lateral ventricles of the brain overflowed with blood,

8) hemorrhages in the quadrangular lobules of the cerebellum, caused by the pressure of the edges of the tentorium on the cerebellum during dislocation of the brain and some others.

In deceased children, as a result of compression of the skull, 4 types of conditions develop: 1) $\mathrm{CH}, 2) \mathrm{BT}, 3) \mathrm{CH}+\mathrm{BT}, 4) \mathrm{BT}+\mathrm{CH}$. In type 1 , the fetus develops $\mathrm{CH}$, accompanied by hypoxia of the brain and its injuries, which leads to the development of complications, death or recovery. In type 2, the fetal head is subjected to severe compression (for example, in pelvic presentation), ruptures occur, massive hemorrhages, leading to death. With type 3, a fetus experiencing $\mathrm{CH}$ in labor increases compression, which leads to the addition of signs of birth trauma. With type 4, the head of the fetus in the absence of $\mathrm{CH}$ immediately begins to experience severe compression (for example, when applying obstetric forceps), which leads to BT, severe hemorrhages, increasing intracranial pressure and leading to impaired cerebral circulation and hypoxia of the brain. With 3 and 4 types of lesions diagnosed with BT.

It should be noted that $\mathrm{CH}$ and $\mathrm{BT}$ are not only often combined, but also often occur on the background of violations of the general hemodynamics of the fetus and general hypoxia. An increase in intrauterine pressure is accompanied not only by the configuration of the head of the fetus, but also by impairment of the uteroplacental circulation and hypoxia of the fetus. For example, severe contractions are accompanied by pronounced contractions of the uterus and can lead to impaired uteroplacental circulation and gas exchange in the placenta. Fast delivery, accompanied by strong and frequent contractions, often a decrease in mother's blood saturation with oxygen, circulatory disorders in the vessels of the placenta, lead to periods of general hypoxia of the fetus and contribute to hypoxic brain damage. Consequently, the degree of configuration of the head reflects the general condition of the fetus, the degree of impaired systemic blood circulation in the fetus, and not only in the brain. Therefore, $\mathrm{CH}$ is often combined with general hypoxia of the fetus, aspiration of amniotic fluid.

With each contraction of the uterus (pain), the intrauterine pressure rises, the fetus's heart rate compensatory decreases and the blood pressure rises. This provides improved blood flow through the placenta and fetal organs, prevents the development of hypoxia. With the end of the pain in the normal everything is restored. Continuing bradycardia between contractions of the uterus indicates that normal circulation has not been restored. An increase in the degree of configuration of the head requires an ever increasing in blood pressure in the fetus in order to restore impaired blood circulation in the brain (as a result of compression of the vessels and sinuses), in the placenta and umbilical cord. This increases the physiological load on the heart. Develop circulatory and hypoxic brain damage. The development of cardiac decompensation and death of the fetus is possible. Similar changes occur during prolonged labor and weak labor.

Thus, fetal hypoxia during labor is caused not only by impairment of the uteroplacental and umbilical cord blood circulation, but also by compression of the head during labor. This type of hypoxia is called $\mathrm{CH}$ and is associated with impaired blood circulation in the pathology of the skull configuration and compression of the head. A child may die in childbirth, after birth, or remain alive. The process of childbirth is accompanied by a configuration of the head, in which the brain moves, changes shape, the veins of the bridge are stretched, the sinuses are compressed, the outflow of venous blood changes, etc. There are 3 degrees of the configuration of the head, and also distinguish the physiological and pathological configurations [7].

In the case of a pathological configuration, hypoxia and acidosis occurs in the brain tissue, there are damages of both hypoxic-ischemic and traumatic nature. How to distinguish between these damages? For BT of the skull are characterized by ruptures of the tentorium cerebelli, falx, bridging veins, subdural and large-focal subarachnoid hemorrhages, intracerebral hemorrhages and other signs. For HIE due to the $\mathrm{CH}$, which is characteristic intradural hemorrhage in the tentorium cerebelli and falx, subarachnoid hemorrhages in the places of origin of the bridge veins, subpial hemorrhages on the apex of the cerebral hemispheres of the brain, subependymal hemorrhages and other. In the case of two nosological forms, other types of hypoxic-ischemic brain lesions may be present: periventricular leucomalacia, selective neuronal necrosis, intraventricular hemorrhages, and other lesions. Thus, fetal hypoxia leads to HIE, and one of its types - compression hypoxia - leads to hypoxicischemic compression encephalopathy.

Conclusion

In the process of labor under the action of mechanical forces and compression of the skull, two types of lesions (conditions, diseases) can occur: birth trauma and compression hypoxia. They differ in the nature of the resulting damage to the skull and brain. In BT, gross mechanical damage dominates in the form of tears, subdural hemorrhages, etc. During $\mathrm{CH}$, hypoxic brain damage caused by impaired blood circulation, venous congestion, increased intracranial pressure, compression of cerebral vessels, etc., dominates. It is necessary to differentiate between two different nosological forms of brain damage in children - BT and $\mathrm{CH}$ brain in children. There are characteristic pathological changes in these pathologies. Clinicians may try to diagnose these changes in living children, pathologists determine morphological changes in the dead. In order to prevent $\mathrm{CH}$ and birth trauma, it is necessary to diagnose the degree of configuration of the head during labor, to make sure that the physiological configuration does not become pathological.

Funding source - Missing.

\section{References}

1. Potter E. (1971) Pathological anatomy of fetuses, newborns and young children. M: Medicine.

2. Lindgren L. (1977) The influence of pressure upon the fetal head during labor. Acta Obstet Gynecol Scand; 56 (4): 303-309.

3. Schifrin B.S, Deymier P.A, Cohen W.R. (2014)Cranial compression ischemic encephalopathy: fetal neurological injury. In: Stress and Developmental Programming: Beyond Phenomenology. Hauppauge, NY: Nova Scientific Publishers; 651-688.

4. Birth injury to the head (basics of personalized medical care). Tutorial. Ed. Jova A.S. St. Petersburg; 2018.

5. Dergachev I.S. (1964) Pathological anatomy and pathogenesis of diseases of newborns, infants and young children. Moscow: Medicine.

6. Gutner M.D. (1958) Intranatal asphyxia. Krasnoyarsk: Medical Institute.

7. Vlasyuk V. V. Birth Trauma and Perinatal Brain Damage. Springer International Publishing, Springer Nature Switzerland AG; 2019.

8. Vlasyuk V.V. (2019) Compression Circulatory Hypoxia of the Brain as a Type of Intrapartum Hypoxia. J Gynecol 4 (1): 000173. 